Documentation et bibliothèques

DOCUMENTATION BIBLIOTHEQQUES

\title{
Modèle de développement d'une bibliothèque virtuelle sur le Web
}

\section{Sylvie Tellier}

Volume 43, numéro 2, avril-juin 1997

Les enjeux et défis de la bibliothèque spécialisée

URI : https://id.erudit.org/iderudit/1033066ar

DOI : https://doi.org/10.7202/1033066ar

Aller au sommaire du numéro

\section{Éditeur(s)}

Association pour l'avancement des sciences et des techniques de la documentation (ASTED)

\section{ISSN}

0315-2340 (imprimé)

2291-8949 (numérique)

Découvrir la revue

\section{Citer cet article}

Tellier, S. (1997). Modèle de développement d'une bibliothèque virtuelle sur le Web. Documentation et bibliothèques, 43(2), 67-72.

https://doi.org/10.7202/1033066ar

Tous droits réservés $@$ Association pour l'avancement des sciences et des techniques de la documentation (ASTED), 1997
Ce document est protégé par la loi sur le droit d'auteur. L'utilisation des services d'Érudit (y compris la reproduction) est assujettie à sa politique d'utilisation que vous pouvez consulter en ligne.

https://apropos.erudit.org/fr/usagers/politique-dutilisation/ 


\title{
Modèle de développement d'une bibliothèque virtuelle sur le Web*
}

\author{
Sylvie Tellier \\ Centre de recherche informatique de Montréal
}

Les spécialistes en information s'inquiétaient de «l'explosion documentaire», cette parution de millions d'ouvrages auxquels il fallait assurer l'accès. Ils doivent à présent composer avec un tout nouveau contexte: la rapide évolution des réseaux télématiques, l'édition électronique et l'avènement d'Internet entraînent la production d'une masse considérable d'informations en dehors des murs de la bibliothèque.

La bibliothèque virtuelle, cette bibliothèque sans murs, entraîne des changements profonds dans le mode de communication entre les fournisseurs d'information et les usagers. Elle permet d'offrir des services documentaires et d'information à distance. Pour les bibliothèques et les centres de documentation spécialisés ${ }^{1}$, elle permet de mettre en commun des ressources provenant du monde entier qui portent sur leur domaine d'intérêt.

Les premières manifestations de bibliothèque virtuelle dans Internet ont fait leur apparition sous deux formes: des collections de documents électroniques stockés sur FTP et pouvant être téléchargés localement et des catalogues collectifs de bibliothèques sur Telnet, également appelés OPAC (Online Public Access Catalog). Mais c'est avec l'avènement du Web et de ses interfaces de navigation que la bibliothèque virtuelle a pris une place véritable sur l'inforoute. De quelques-unes il a deux ans, elles sont passées à plusieurs dizaines, car les technologies pour les mettre sur pied sont facilement accessibles.

La masse considérable d'informations déposées dans Internet peut devenir une ressource de première importance pour les services d'information spécialisés à la condition qu'elle soit sélec- tionnée et organisée en fonction des besoins spécifiques des usagers. Or, si la bibliothèque virtuelle peut devenir un lieu privilégié pour organiser l'information, si les technologies pour la développer sont disponibles, il est peu question de sa «modélisation». Les bibliothèques de ce type sur le Web peuvent servir de modèle mais elles sont souvent créées par de grosses organisations. Le modèle proposé ici, bien que générique, s'adresse tout particulièrement aux services d'information spécialisés désirant mettre sur pied une bibliothèque virtuelle.

\section{Définition}

La confusion entourant le concept de bibliothèque virtuelle vient de la diversité des appellations: les expressions "bibliothèque virtuelle», «bibliothèque électronique» et «bibliothèque numérisée» sont souvent employées indifféremment. La bibliothèque virtuelle est une bibliothèque "sans murs" dont la collection et les services sont mis en réseau et partagés avec les usagers et avec d'autres services. II faut distinguer la bibliothèque virtuelle de la bibliothèque électronique, concept davantage axé sur le support des documents. Ainsi, une bibliothèque mettant des ordinateurs et leurs périphériques (par exemple des lecteurs de disques optiques) à la disposition de sa clientèle est une bibliothèque électronique. La bibliothèque virtuelle peut cependant comprendre le concept de bibliothèque numérisée, axé sur une collection de documents à l'origine sous des formats traditionnels mais numérisée à l'aide des nouvelles techniques de reproduction. Les projets de numérisation sont très souvent réalisés par d'importantes bibliothèques car ils sont coûteux et de longue haleine. Du côté des services d'information spécialisés, comme celui du Centre de recherche informatique de
Montréal (CRIM), la collection s'enrichit de documents électroniques de divers formats (HTML, Postcript, ASCII, etc.) repérés sur Internet, organisés par thèmes et par types dans des pages Web. Les collections de bibliothèques entièrement virtuelles, c'est-à-dire sans équivalent physique, ne se composent d'ailleurs que de documents Internet, comme la WWW Virtual Library.

\section{Modèle de développement}

Le modèle de développement d'une bibliothèque virtuelle proposé ici s'inspire largement de la méthodologie d'élaboration d'un site Web qui en est le support. Les étapes, détaillées dans le tableau 1 , en sont la conceptualisation, la planification, le développement proprement dit, la validation et l'exploitation.

\section{La conceptualisation}

Le désir de mettre sur pied une bibliothèque virtuelle doit avant tout être guidé par la connaissance de ce qu'elle est et de ce qu'elle implique. Une documentation volumineuse et diversifiée peut être trouvée dans les banques de données commerciales, comme INSPEC et LISA et sur Internet. La consultation d'experts peut aussi contribuer à cette prise de connaissance: au Québec, on les trouve dans certains organismes où des bibliothèques virtuelles ont été créées, notamment dans des universités, dans des centres de recherche (comme le CRIM) et dans des bibliothèques publiques. Enfin,

* Certaines parties de cet article s'inspirent du cours "Design de sites Web" donné par le Service de formation du Centre de recherche informatique de Montréal.

1. Ci-après dénommés services d'information spécialisés. 

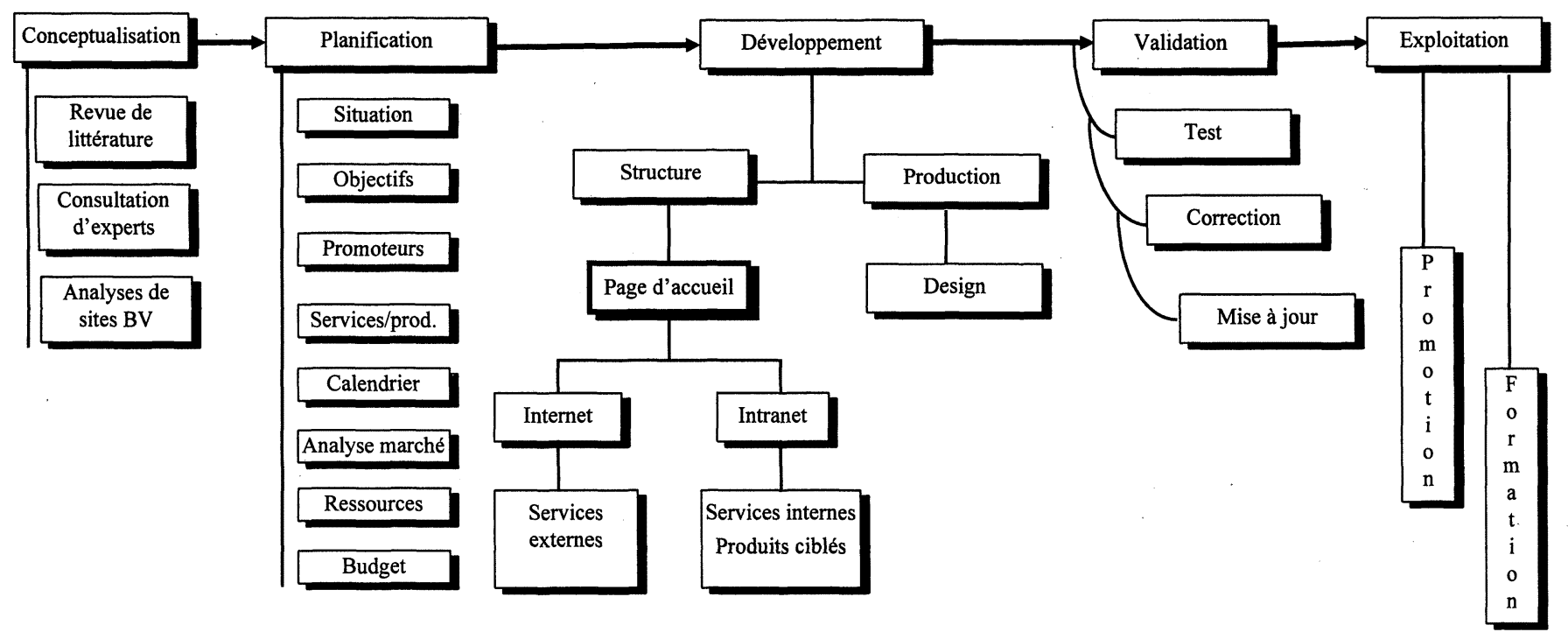

la visite de bibliothèques virtuelles sur le Web permettra de préciser l'idée souvent abstraite que l'on s'en fait.

\section{La planification}

La décision d'implanter une bibliothèque sans murs est fonction du milieu qu'elle desservira. Un projet de bibliothèque virtuelle doit commencer par l'étape cruciale d'élaboration d'un plan de développement lequel implique l'examen de la situation, la définition d'objectifs, l'identification des promoteurs, le choix de services et produits, l'établissement d'un calendrier de réalisations, l'analyse du marché, l'identification des ressources nécessaires et enfin, les prévisions budgétaires. Pour chacun de ces paramètres, plusieurs questions doivent être posées. En voici une liste partielle présentée sous forme de tableau (tableau 2).

Pour répondre à ces questions voici quelques considérations touchant plus particulièrement les services, les ressources humaines et financières.

\section{Services}

Dans les centres de documentation et les bibliothèques spécialisées, les membres du personnel sont généralement en petit nombre, ce qui peut freiner la volonté ou la possibilité de développer une bibliothèque virtuelle. En ce cas, il faut choisir d'y intégrer des services et des produits qui allégeront éventuellement le travail du personnel. Le CRIM, par exemple, est à mettre en place un service d'achat sur son intranet qui minimisera l'intervention du centre de documentation dans l'acquisition de documents. II faut aussi songer à des services, comme l'accès à une collection de documents, qui apporteront une plus-value aux services déjà existants.

\section{Choix de l'information}

L'information sur Internet est à ce point volumineuse que son évaluation est essentielle à une sélection éclairée. La grande différence entre le document imprimé et le document Web est que dans le deuxième cas, la publication se fait souvent sans éditeur. Dans les deux cas, on retrouve des documents grand public et des documents scientifiques, mais sur l'inforoute des documents de type publicitaire ainsi que des documents multimédias s'y ajoutent.

Dans un système d'information traditionnel, une politique de choix de documents écrite ou implicite est mise en application. Dans le contexte de la bibliothèque virtuelle, cette politique devrait te- nir compte des documents Web. En effet, la bibliothèque virtuelle sera non seulement constituée de documents numérisés mais aussi de nombreux pointeurs vers des pages Web qui constituent également des documents.

Voici quelques critères que devrait satisfaire un document Web pour qu'il puisse être intégré dans une bibliothèque virtuelle: stabilité de l'information, autorité de l'auteur, compatibilité du format, comparaison avec d'autres ressources, exhaustivité de l'information, présentation du site, organisation de l'information, facilité d'accès aux ressources, mise à jour de l'information.

\section{Acquisition}

Les délais d'acquisition sont souvent une pierre d'achoppement dans l'évaluation de services d'information. Le Web atténue ce problème puisqu'il met à disposition les catalogues d'éditeurs, des listes de prix et des formulaires d'achat. II permet de plus l'accès partagé à des ressources d'information électroniques (bulletins électroniques), ce qui minimise les coûts d'acquisition.

\section{Traitement}

La bibliothèque virtuelle ne se définit pas seulement comme un service à 


\begin{tabular}{|c|c|}
\hline $\begin{array}{l}\text { Éléments du plan de } \\
\text { développement }\end{array}$ & Tableau 2. Questions types \\
\hline Situation actuelle & $\begin{array}{l}\Rightarrow \text { Quels sont les services offerts? } \\
\Rightarrow \text { Quel est l'état de la technologie? } \\
\Rightarrow \text { Quels sont les outils utilisés? } \\
\Rightarrow \text { Quel est le mode habituel de diffusion d'information et qui en sont les } \\
\Rightarrow \text { responsables? } \\
\Rightarrow \text { Le personnel et la clientèle sont-ils aptes à accepter un changement? } \\
\Rightarrow \text { Quelle est la clientèle actuelle? Connaît-elle Internet? } \\
\Rightarrow \text { Comment cela affectera-t-il le service actuel (personnel, services)? }\end{array}$ \\
\hline Objectifs & $\begin{array}{l}\Rightarrow \text { Pourquoi implanter une bibliothèque virtuelle? } \\
\Rightarrow \text { L'implantation répond-elle aux besoins du milieu? } \\
\Rightarrow \text { Quel en est le profit pour mon organisation? } \\
\Rightarrow \text { Permettra-t-elle au service de se mettre à la pointe des nouvelles } \\
\Rightarrow \text { technologies et méthodes de communication? } \\
\Rightarrow \text { La BV assurera-t-elle une plus grande visibilité du service déjà en place? }\end{array}$ \\
\hline Promoteurs & $\begin{array}{l}\Rightarrow \text { Le projet a-t-il l'appui de la direction? } \\
\Rightarrow \quad \text { Peut-il impliquer des partenaires externes? }\end{array}$ \\
\hline Services et produits & $\begin{array}{l}\Rightarrow \text { Quels sont les domaines couverts par la bibliothèque virtuelle? } \\
\Rightarrow \text { Le projet implique-t-il la numérisation de la collection? } \\
\Rightarrow \text { Les techniques d'organisation et d'accès à l'information sont-elles } \\
\Rightarrow \text { maîtrisées? } \\
\Rightarrow \text { Le projet prévoit-il un partage des ressources avec d'autres organismes? } \\
\Rightarrow \text { Veut-on développer des services internes et des services externes? } \\
\Rightarrow \text { Quels sont les services/produits que l'on veut fournir? } \\
\Rightarrow \text { Veut-on développer un intranet? }\end{array}$ \\
\hline Calendrier de réalisations & $\begin{array}{l}\Rightarrow \text { De combien de temps dispose-t-on pour réaliser le projet? } \\
\Rightarrow \text { Quelle est la durée de chaque étape? } \\
\Rightarrow \text { Quelle est le temps requis pour développer chaque service/produit? }\end{array}$ \\
\hline Analyse de marché & $\begin{array}{l}\Rightarrow \text { La BV permettra-t-elle de rejoindre une clientèle qui ne le serait pas } \\
\text { autrement? } \\
\Rightarrow \quad \text { Un sondage a-t-il été réalisé pour identifier les besoins de la clientèle? }\end{array}$ \\
\hline Ressources & $\begin{array}{l}\Rightarrow \text { Le projet peut-il compter sur l'appui d'un service informatique? } \\
\Rightarrow \text { Le personnel autre que les spécialistes en information peut être mis à } \\
\text { contribution? } \\
\Rightarrow \quad \text { Y a-t-il déjà dans l'organisme des personnes expérimentées dans le } \\
\text { développement de sites Web? } \\
\Rightarrow \text { Quel matériel sera nécessaire? }\end{array}$ \\
\hline Budget & $\begin{array}{l}\Rightarrow \text { Quelles sont les dépenses engendrées par les salaires, le matériel, les frais } \\
\text { généraux fixes, etc.? } \\
\Rightarrow \text { Des revenus sont-ils envisagés? }\end{array}$ \\
\hline
\end{tabular}


l'usager. Elle est d'abord et avant tout affaire d'accès à l'information, information dont la prolifération exige la description et l'organisation.

L'usage d'Internet pour le catalogage de ressources d'information favorise le travail de collaboration entre établissements de même type. Les indicateurs de succès dans un projet de ce genre sont le degré de participation d'un nombre significatif de services d'information documentaires et la qualité du catalogage. Pour s'assurer du respect des normes, l'organisme responsable doit établir un manuel de catalogage qui pourra être adopté par tous les organismes ou départements participant au programme.

Par ailleurs, le Web permet aux responsables du traitement de trouver de l'information sur des sujets peu connus, d'avoir accès à plusieurs outils de catalogage en ligne (par exemple, la liste de vedettes-matière de la Library of Congress), d'ajouter de l'information complémentaire au catalogue (par exemple, les tables des matières), de copier et de coller des données prises directement du Web.

\section{Référence}

Dans le monde des bibliothèques virtuelles, les contacts interpersonnels sont d'autant plus importants que le service est complètement dépersonnalisé. Demander à l'usager de s'inscrire et d'indiquer par la même occasion son profil d'intérêt, lui faire parvenir un bulletin d'information, lui offrir une liste de suggestions de lecture ainsi qu'un catalogue d'ouvrages qu'il peut emprunter sont autant de moyens de lui faire sentir que les services lui sont destinés.

Un service "question/réponse» peut aussi être offert aux usagers. II est cependant prudent de limiter ce service à une clientèle cible, car la communauté Internet est beaucoup trop vaste pour être desservie par un personnel limité.

Certains services peuvent être tarifés. Au CRIM, les services de veille portant sur les technologies de l'information sont accessibles par abonnement sur un extranet.

\section{Ressources humaines}

Le personnel des bibliothèques n'est pas remplacé par l'ordinateur. L'apparition du Web n'en diminue certainement pas le rôle. Au contraire, l'importance des spécialistes de l'information dans l'apport de valeur ajoutée à l'information déposée dans Internet est de plus en plus soulignée dans la littérature professionnelle. II est vrai cependant que le rôle et les tâches du personnel sont appelés à changer: apprendre le codage HTML, monter un site, le gérer, organiser l'information électronique sont autant de nouvelles tâches. Mais la bibliothèque virtuelle ne remplace pas la bibliothèque traditionnelle: aux tâches traditionnelles reliées à l'information imprimée s'ajoutent les tâches reliées à l'information électronique. Bien qu'il soit important de faire participer tout le personnel au développement de la bibliothèque virtuelle, il faut $y$ affecter une personne à temps plein pendant la période de développement ${ }^{2}$, puis à temps partiel pour la mise à jour continue.

Outre le personnel de la bibliothèque, il faut prévoir une équipe de personnes plus ou moins grande selon l'ampleur du site: gestionnaire de projet, responsable éditorial, graphiste, concepteur, programmeur (HTML, Java, Perl, VRML, etc.), administrateur de serveur, correcteur, traducteur, gestionnaire du site, formateur, etc. Pour une partie ou l'ensemble du développement, on peut envisager la sous-traitance. En ce cas, il est primordial que le personnel en place soit partie prenante.

\section{Budget}

Malgré l'apparente facilité de constitution d'un site Web, une bibliothèque virtuelle peut rapidement prendre des proportions inattendues et sa mise à jour devenir fastidieuse. Autrement dit, il est beaucoup plus coûteux et exigeant qu'il n'en paraît au départ. Selon Gartner Group, les coûts de développement d'un site Web varient entre 104000 \$ et $285000 \$$, auxquels des coûts annuels de $49000 \$$ à $111000 \$$ s'ajoutent. Le développement d'un petit site peut coûter entre 20000 \$ et $50000 \$^{3}$

\section{Le développement}

Le développement proprement dit d'un site Web consiste à définir la structure du site puis à en faire la production. Bien qu'une bibliothèque virtuelle produite par un service d'information spécialisé puisse être de petite envergure, sa structure devrait tout de même se composer de deux volets: l'un sur Internet et l'autre sur un intranet. En effet certains services, tels les services techniques, sont des services internes qui ne concernent que l'organisme auquel le service d'information est affilié; par exemple, la liste des emprunteurs n'a pas à être diffusée à l'extérieur de l'organisme. Dans d'autres cas, les services internes ne concernent que le personnel responsable; par exemple, les formulaires d'achat et les budgets n'ont pas à être diffusés ni à l'extérieur ni à l'intérieur de l'organisme. Dans ce cas, il importe de considérer la constitution d'un intranet limitant l'accès par mot de passe à certains usagers de la bibliothèque virtuelle.

Le tableau 3 propose des éléments pouvant faire partie d'une bibliothèque virtuelle avec la mention du mode de diffusion (Internet ou intranet). Le choix de l'un ou de l'autre mode de diffusion dépend évidemment des politiques et des façons de faire du milieu dans lequel la bibliothèque virtuelle est implantée.

\section{La validation}

L'étape de validation est cruciale puisqu'elle garantit la qualité de la bibliothèque virtuelle mise en place. Elle comprend un test «d'utilisabilité» permettant de vérifier auprès d'usagers la fonctionnalité du site, c'est-à-dire la pertinence de l'organisation de l'information et de ses accès. On y prévoit également une activité constante de mise à jour. Pour réaliser le test d'utilisabilité, il faut suivre le cheminement suivant: définir les buts du test, ce

2. La durée de cette période dépend de l'ampleur du projet, mais aussi des connaissances et du savoir-faire de la personne désignée au développement.

3. Cité dans le cours «Design de sites Web» du Service de formation du Centre de recherche informatique de Montréal 
Tableau 3. Liste d'éléments pouvant faire partie d'une bibliothèque virtuelle

\begin{tabular}{|c|c|c|c|}
\hline Types & Éléments & Internet & Intranet \\
\hline \multirow[t]{3}{*}{ Coordonnées } & Nom et adresse du lieu physique de la bibliothèque & $x$ & \\
\hline & Numéros de téléphone par département ou filiale & $x$ & $\mathrm{x}$ \\
\hline & Liste de tous les employés & $x$ & $x$ \\
\hline \multirow[t]{6}{*}{ Présentation } & Message du responsable & $x$ & \\
\hline & Mission & $\mathrm{x}$ & \\
\hline & Histoire de l'établissement et plan de développement & $\mathrm{x}$ & \\
\hline & Liste des départements et filiales & $\mathrm{x}$ & \\
\hline & Activités à venir & $x$ & \\
\hline & Région et clientèle desservies & $x$ & \\
\hline Accès & $\begin{array}{l}\text { Heures d'ouverture } \\
\text { Plan de la bibliothèque }\end{array}$ & & \\
\hline \multirow[t]{2}{*}{ Services } & Liste de services & $x$ & \\
\hline & Adresses électroniques du personnel par service & $x$ & \\
\hline \multirow[t]{3}{*}{ - Acquisition } & Liste de nouvelles acquisitions organisées par sujet & $x$ & \\
\hline & Formulaires de suggestions d'achat & & $x$ \\
\hline & Formulaires d'achat & & $x$ \\
\hline \multirow[t]{4}{*}{ - Prêt } & Périodes de prêt & & $x$ \\
\hline & Méthodes de retours & & $\mathrm{x}$ \\
\hline & Conditions si retard ou non-retour & & $x$ \\
\hline & Liste de documents empruntés & & $x$ \\
\hline \multirow{3}{*}{ - Accès à la collection } & Consultation du catalogue + guide & $\mathrm{x}$ & \\
\hline & Bibliographies thématiques & $x$ & \\
\hline & Autres sites organisés par thèmes & $x$ & \\
\hline \multirow[t]{7}{*}{ - Référence } & Foire aux questions & $x$ & \\
\hline & Index-sujet à des ressources du Web & $x$ & \\
\hline & Formulaire de questions de référence & & $\mathrm{x}$ \\
\hline & Comment chercher sur le Web? & $\mathrm{x}$ & \\
\hline & Liste limitée d'outils classés par type de ressources & $\mathrm{x}$ & \\
\hline & Suggestions de lecture & $x$ & \\
\hline & Groupes d'intérêt sur certains sujets & & $x$ \\
\hline \multirow[t]{3}{*}{ Divers } & Autres bibliothèques virtuelles et sites associés & $\mathrm{x}$ & \\
\hline & Formulaire de questions et de commentaires sur le site & $x$ & \\
\hline & URL et date de mise à jour & $x$ & \\
\hline
\end{tabular}


que l'on veut vérifier; identifier les participants (personnes étrangères au processus de développement) et les recruter; sélectionner et organiser les tâches à tester; identifier les critères (nombre d'erreurs, facilité d'utilisation, satisfaction, temps d'apprentissage, etc.); décider comment mesurer «l'utilisabilité» (pondération, cueillette de commentaires, etc.); préparer le test et le conduire; analyser les résultats; recommander des changements et apporter les corrections.

\section{L'exploitation}

L'étape d'exploitation est une étape cruciale puisque, sans elle, il n'est pas garanti que le site de la bibliothèque virtuelle soit visité, si ce n'est par le fait du hasard, et utilisé adéquatement.

\section{Promotion de la bibliothèque vir- tuelle}

Un site Web n'en n'est qu'un parmi des milliers d'autres. II ne faut donc pas hésiter à faire la promotion de la bibliothèque virtuelle. II existe plusieurs moyens d'assurer sa visibilité: s'inscrire aux différents outils du Web; participer aux groupes d'intérêt et, mieux encore, en former un; publier un bulletin destiné aux usagers et compiler des bibliographies spécialisées, etc.

\section{Formation}

Pour exploiter pleinement la bibliothèque virtuelle et plus largement Internet, il faut prévoir des séances de formation pour l'ensemble du personnel du service d'information. Les cours à conseiller sont l'introduction à Internet, la recherche d'information sur Internet et la conception d'un site Web.

Pour en assurer une meilleure utilisation, il faut aussi prévoir des séances de formation pour les usagers du service d'information. De plus, la bibliothèque virtuelle pouvant attirer de nouveaux usagers, il faut considérer également l'intégration d'un tutoriel dans le site Web de la bibliothèque virtuelle.

\section{Conclusion}

Le monde des bibliothèques virtuelles est fascinant. II est en pleine expan- sion et on y sent encore un grand esprit de liberté. Tout le monde peut s'y lancer, les règles n'y étant pas encore clairement établies.

Les bibliothèques virtuelles ont d'abord été l'apanage de grosses organisations mais elles peuvent indéniablement servir les bibliothèques et centres de documentation spécialisés aussi bien pour l'accessibilité à leurs collections et services que pour l'administration de leurs activités sur un intranet. Mais il est temps d'en modéliser le développement afin de permettre aux nouveaux venus d'éviter les tâtonnements de leurs prédécesseurs. Les responsables des services d'information spécialisés, tentés de s'y aventurer, se posent certainement de nombreuses questions sur le bien-fondé d'une bibliothèque virtuelle. Nous n'avons pas tenté de répondre à toutes leurs interrogations mais de les orienter vers les bonnes questions.

\section{Bibliographie}

American National Standard Institute. ANSI Z39.50: Information retrieval service and Version 2. $1992.42 \mathrm{p}$.
Dumas, Joseph S. and Janice C. Redish. A practical guide to usability testing. Norwood, NJ: Ablex, 1993. $412 \mathrm{p}$.

Harden, Sheila and Robert Harden. Net notions for librarians. http://dspace.dial.pipex.com/town/ square/ac $940 /$ netnotes.html

Krumenaker, Larry. Virtual libraries, with journals, get real. Science vol. 260 (21 may 1993): 10661067.

Kurzweil, Raymond. «The virtual library». Library journal (15 March 1993): 54-55.

Lenzini, Rebecca T. and Ward Shaw. «Creating a new definition of library cooperation: Past, present, and future models». Library administration and management (Winter 1991) : 37-40.

Gell Mason, Marilyn. «Library automation: The next wave». Library administration and management (Winter 1991): 34-37.

Stewart, Barbara. Top 200 technical services benefits of home page development. Amherst: University of Massachussetts, 1996. http:/ tpot.ucsd.edu/Cataloging/Misc/top200.html

Sylvan, Peter. Web page design guidelines for public libraries. Milton: Milton Public Library,1996. http:/www.tiac.net/users/mpl/ guidelines.html

\begin{tabular}{|c|c|}
\hline \multicolumn{2}{|c|}{$\begin{array}{l}\text { L'index mensuel de la Gazette officielle du Québec II } \\
\text { CIna faÇOnS }\end{array}$} \\
\hline \multirow{2}{*}{\multicolumn{2}{|c|}{$\begin{array}{l}\text { "L'index analytique permet de ne rien ignorer des décisions } \\
\text { gouvernementales en matière de loi et de règlement." } \\
\qquad \text { Monique Dumont, éditrice et indexeure } \\
\text { Abonnement mensuel cumulatif unique en son genre }\end{array}$}} \\
\hline & \\
\hline ABONNEZ-VOUS MAINTENANTI & Cartable inclus \\
\hline Nom: & Prix (1 an): $78,00 \$$ \\
\hline Adresse: & $\begin{array}{ll}\text { TPS: } & 5,46 \$ \\
\text { TVQ: } & 5,42 \$ \\
\end{array}$ \\
\hline Ville: & Total: $88,88 \mathrm{~S}$ \\
\hline Province: & Mode de paiement \\
\hline $\begin{array}{l}\text { Signature: } \\
\text { Téléphone: }\end{array}$ & $\begin{array}{l}\square \text { Chèque } \\
\square \text { Mandat-poste }\end{array}$ \\
\hline 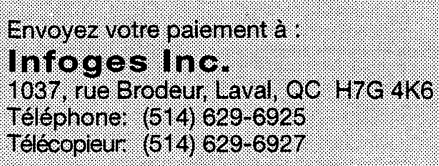 & $\begin{array}{l}\text { Format désiré } \\
\square \text { coumier } \\
\square \text { téécopieur } \\
\square \text { disquette WordPerfect } \\
\square \text { courrier électronique }\end{array}$ \\
\hline
\end{tabular}

